\title{
Modeling wind-buffeting of the Thirty Meter Telescope
}

Douglas G. MacMynowski, Carl Blaurock, George Z. Angeli, Konstantinos Vogiatzis

Douglas G. MacMynowski, Carl Blaurock, George Z. Angeli, Konstantinos Vogiatzis, "Modeling wind-buffeting of the Thirty Meter Telescope," Proc. SPIE 6271, Modeling, Systems Engineering, and Project Management for Astronomy II, 62710M (22 June 2006); doi: 10.1117/12.672706

SPIE Event: SPIE Astronomical Telescopes + Instrumentation, 2006, Orlando, Florida, United States 


\title{
Modeling wind-buffeting of the Thirty Meter Telescope
}

\author{
Douglas G. MacMynowski ${ }^{a}$, Carl Blaurock ${ }^{b}$, George Z. Angeli ${ }^{c}$, and Konstantinos Vogiatzis ${ }^{d}$ \\ ${ }^{a}$ California Institute of Technology \\ Department of Control and Dynamical Systems, Pasadena CA 91125 \\ ${ }^{b}$ NightSky Systems Inc., Raleigh, NC 27616 \\ ${ }^{c}$ TMT Project Office, Pasadena, CA 91125 \\ ${ }^{d}$ AURA New Initiatives Office, NOAO, Tucson, AZ 85719
}

\begin{abstract}
The Thirty Meter Telescope project is designing a $30 \mathrm{~m}$ diameter ground-based optical telescope. Unsteady wind loads on the telescope structure due to turbulence inside the telescope enclosure impact the delivered image quality. A parametric model is described that predicts the optical performance due to wind with sufficient accuracy to inform relevant design decisions, including control bandwidths. The model is designed to be sufficiently computationally efficient to allow rapid exploration of the impact of design parameters or uncertain/variable input parameters, and includes (i) a parametric wind model, (ii) a detailed structural dynamic model derived from a finite element model, (iii) a linear optical response model, and (iv) a control model. Model predictions with the TMT structural design are presented, including the parametric variation of performance with external wind speed, desired wind speed across the primary mirror, and optical guide loop bandwidth. For the median mountaintop wind speed of $5.5 \mathrm{~m} / \mathrm{s}$, the combination of dome shielding, minimized cross-sectional area, and control results in acceptable image degradation.
\end{abstract}

\section{INTRODUCTION}

The Thirty Meter Telescope (TMT) ${ }^{1}$ conceptual design is shown in Fig. 1. Several other design studies for large ground-based optical telescopes are also underway. ${ }^{2-4}$ One of the factors that contributes to the delivered image quality is the image motion and image blur resulting from uncorrected telescope vibrations caused by turbulent wind inside the telescope enclosure. The response depends on multiple design choices. Characterizing the wind loads inside the enclosure and the optical consequences that result is thus essential during the design stage in order to inform relevant design decisions, and to allocate an appropriate contribution to the telescope error budget.

Significant progress has been made by the TMT team in understanding the wind environment through telescope measurements, ${ }^{5-7}$ wind-tunnel testing, ${ }^{8,9}$ computational fluid dynamics (CFD) ${ }^{10}$ and the integration of these sources of information into a coherent parametric wind model. ${ }^{11,12}$ Progress has also been made in modeling the dynamic response of the telescope structure to the wind disturbance. ${ }^{12,13}$ Similar efforts in understanding the wind and the telescope response have been made by other design teams. ${ }^{14}$

The fundamental purpose of any model is to inform particular design decisions. Key decisions that influence the wind response of the telescope include control bandwidths of the telescope main axes (particularly the elevation axis) and optical guiding loops, constraints on the projected area of the secondary mirror support structure, and the desired air flow over the primary mirror that mitigates thermal seeing. The latter leads to a design trade-off to choose the target air flow that minimizes the combined image degradation due to both thermal seeing and wind buffeting. This trade is not explicitly considered herein, but follows from this modeling and thermal modeling. ${ }^{15}$

The modeling strategy should flow from the questions that the model is intended to answer, and a strategy appropriate to high fidelity performance assessment of a particular point design is not necessarily ideal for evaluating the impact of a range of design parameters. It is essential to choose an approach that permits variation of the parameters of interest. Thus, for example, the wind loads herein are parameterized following Ref. 11 rather than directly applying loads obtained from CFD or wind tunnel data as in Refs. 14,16,17. Maintaining sufficient computational simplicity to allow rapid iterations is also important. Finally, the fidelity of the calculations should be appropriate to both the fidelity of the input knowledge and the prediction accuracy required to make design decisions. Thus, for example, the approximation error in using a linear optical model ${ }^{18}$ is small compared to errors in the wind input knowledge or uncertainty in the design details of the ultimate structure, and a full

Modeling, Systems Engineering, and Project Management for Astronomy II,

edited by Martin J. Cullum, George Z. Angeli, Proc. of SPIE Vol. 6271 ,

62710M, (2006) -0277-786X/06/\$15 d doi: 10.1117/12.672706

Proc. of SPIE Vol. 6271 62710M-1 

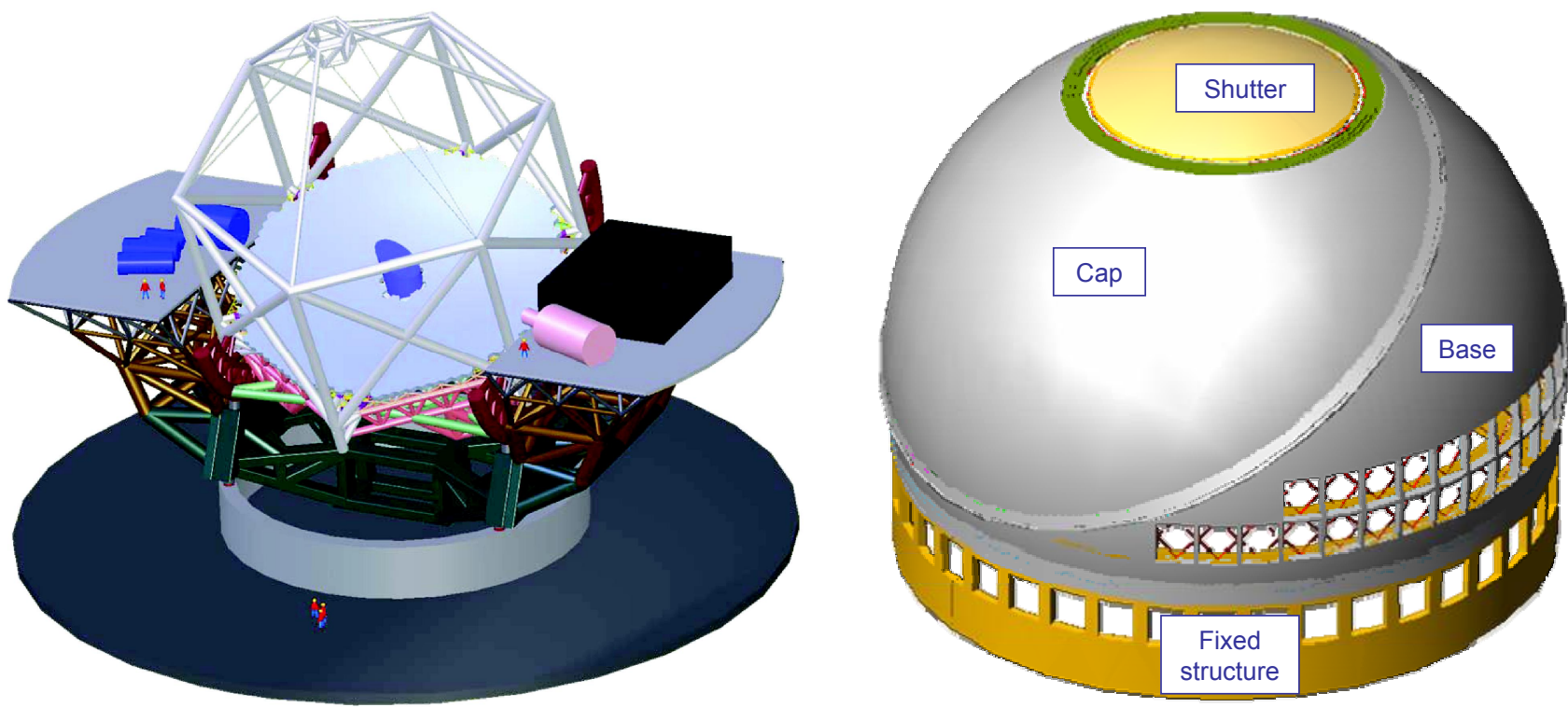

Figure 1. Thirty Meter Telescope (TMT) f/1 design (left) and vented Calotte dome (right). The secondary mirror is Gregorian, and a tertiary mirror at the elevation axis (in front of M1) feeds instruments on Nasmyth platforms. The Calotte enclosure is shown with $100 \%$ of the potential vented area open.

nonlinear optical ray-trace is not appropriate for design trades. The modeling strategy must also evolve with the project as the relevant design questions change, and a flexible code that allows one to progress to more detailed questions is useful. The initial emphasis is on pointing (image motion), then the seeing-limited image blur, then eventually to (high wavenumber) inter-segment edge discontinuities relevant to high-contrast adaptive optics operation. As the telescope design progresses, higher fidelity performance predictions will also become more important to verify the design.

The overall structure of the model is described in Section 2 and shown in Figure 2. A parametric wind model, described in Section 4 is applied to the finite-element derived structural dynamic model, described in Section 3. The structural model is connected to a linear optical model, described briefly in Sec. 5, to compute the optical consequences of structural deformation. Control (section 6) is applied to the telescope main axes, including a low bandwidth optical guiding loop, and also to the primary mirror segment actuators. The performance of the TMT design is described in detail in Sec. 7, including both nominal performance, and the parametric variation of performance with both relevant design parameters such as control bandwidths, and uncertain or variable input parameters such as external wind speed. Future improvements to the model, for example to predict AO-relevant performance, are described in the conclusions.

Three key design decisions lead to acceptable wind performance: (i) choice of dome design with minimum open area to minimize wind loads on M2, (ii) choice of a tripod M2 upper support structure to minimize cross-sectional area, and (iii) optical tip/tilt feedback to the mount control system.

\subsection{Thirty Meter Telescope}

\section{MODEL STRUCTURE}

The Thirty Meter Telescope design is shown in Fig. 1 and described in Ref. 1. A brief discussion follows of design details that affect the wind loads and the telescope response. The f/1 $30 \mathrm{~m}$ diameter primary mirror (M1) is comprised of 738 individual hexagonal segments, with the "out-of-plane" motion controlled by 3 actuators behind each mirror and measured by inter-segment sensors that measure relative displacement (see Refs. 13, 19, 20). The Gregorian secondary mirror (M2) is $3.7 \mathrm{~m}$ in diameter, $30 \mathrm{~m}$ above the elevation axis, and supported by a tripod support structure more similar to existing radio telescopes rather than the spider-supported secondaries common in optical telescopes; this decision was taken in order to reduce the cross-section to wind. All instruments are on Nasmyth platforms, fed by a tertiary mirror (M3) at the elevation axis. The baseline design includes relatively 
fast (fraction of $1 \mathrm{~Hz}$ ) optical guiding via the elevation and azimuth drive control systems, and only slow gravity/thermal compensation for the positions of M2 and M3; this choice is based in large part on the modeling results herein. The control loops are discussed in more detail in Ref. 13.

The telescope enclosure is a Calotte ${ }^{21,22}$ with a circular aperture to minimize open area and therefore wind loads on M2 and its supporting structure. However, some air flow through the enclosure is necessary to mitigate thermal seeing through convective transport of heat away from the primary mirror. It is therefore likely that the enclosure will include vents (as shown in Fig. 1) that can be opened to allow natural ventilation across M1 in low to moderate external wind conditions. A companion modeling study is being undertaken to estimate the air flow required and air flow achievable through vents. ${ }^{15}$ The goal, of course, is to choose adequate venting to minimize the combined image degradation due to both thermal seeing (more venting is better) and wind buffeting (less venting is better), and the venting design must therefore follow from both modeling efforts.

\subsection{Modeling Strategy and Overview}

As noted in the introduction, the purpose of the model is to address relevant design decisions, and the key features of the model that follow from this objective are (i) the ability to vary relevant parameters, either to explore design space, or to explore the effects of unknown or varying external parameters, and (ii) sufficient computational efficiency to practically allow this exploration. The latter requirement leads to several simplifying assumptions, which are frequently different from the approaches taken by other telescope modeling efforts that focus instead on the evaluation of a particular choice of design parameters.
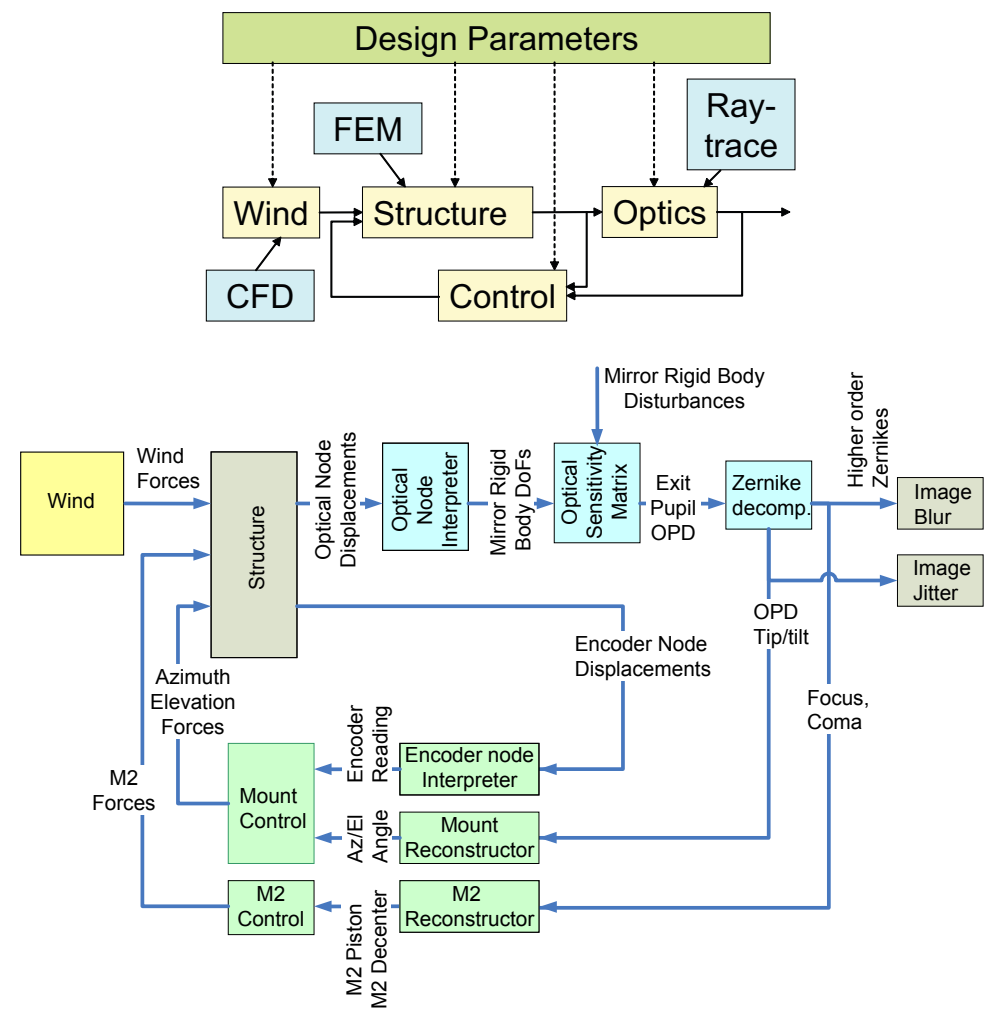

Figure 2. Information flow in parametric model, overview (top) and details on control and optics flow (bottom). Separate FEM and raytrace codes are used offline to produce the structure and optical models, and the wind model is informed by off-line CFD analyses. Each of the wind, structure, optics and controls blocks are influenced by selected design parameters.
Several strategies have been employed to minimize computational effort while retaining useful and meaningful accuracy. The most significant of these is to perform computations in the frequency domain (although the capability also exists for time domain or state space (Lyapunov) performance calculations). This requires linearity, and thus the use of a linear optical model is required. A second broad area in which significant computational savings are possible is in the treatment of the primary mirror segments and their actuators, because the high segment count can easily lead to significant computations. In this regard, it is essential to ask what modeling tools are relevant to which design questions, since it is frequently simpler to design separate tools for separate tasks rather than attempting to design a single model that is capable of answering every question that may arise.

Each segment is supported on actuators and whiffle-trees whose combined stiffness leads to rotational segment support resonances near $35 \mathrm{~Hz}$. The coupling of these resonances through the structure leads to a spread in resonant frequencies. ${ }^{13}$ However, while these structural resonances limit the achievable bandwidth of the primary mirror control system, there is insufficient wind energy at these frequencies for these resonances to contribute significantly to the telescope 
dynamic response. Therefore, rather than designing a single model that can simultaneously estimate the controlstructure interaction with the entire primary mirror control system while also estimating the wind-induced buffeting of the structure, it is far more efficient to separate these tasks and design one model that can assess the achievable control bandwidth, and a separate model that can estimate the wind response. Doing so allows a significant reduction in the number of structural modes that must be used to compute a sufficiently accurate performance estimate, since the dynamics of the segment resonances do not need to be included. (Their static contribution to the deflection does need to be included.) Similarly, the segment control system does not need to be simulated in detail, since the transformation from segment motion to edge sensor response and back to estimated segment motion is well understood, and the resulting sensor noise contribution to image quality can be separately estimated. ${ }^{19,20}$ Finally, while the dynamic characteristics of high spatial wavenumber errors due to relative displacement between neighbouring segments are essential to understand for the prediction of highcontrast adaptive optics (AO) performance, it is not necessarily useful to attempt to design a single modeling tool that simultaneously predicts both the low wavenumber motion readily correctable by $\mathrm{AO}$ and the high wavenumber motion. The latter is dominated by the compliance of individual segment supports rather than the mirror cell (Fig. 3), and there is no reason to include the full structural dynamic model when predicting it.

The overall structure of the model is shown in Fig. 2, where each block on the upper figure represents separate code. This allows both separate verification, and rapid updating, for example with a new telescope finite element model (FEM). The chosen parameters are fed into each of the structure, wind, control, and optics blocks to create an overall system whose performance can be analyzed. While the total number of parameters that can in principle be changed is large, the number that are useful to change is relatively small; these are illustrated through Table 1 and the parametric dependency plots in Section 7. The system objects are defined and performance calculations conducted using the DOCS Matlab ${ }^{\mathrm{TM}}$ toolbox from Nightsky Systems Inc.

\section{TELESCOPE STRUCTURE}

A finite element model of the TMT design has been constructed. If the drives are locked, then for a $30^{\circ}$ zenith angle the first resonant frequency is at roughly $3.7 \mathrm{~Hz}$, involving primarily tilting of the primary mirror and decentering of M2. There are nearly 500 modes below $30 \mathrm{~Hz}$, as shown in Figure 3. A modal representation of the ANSYS model is obtained, converted to Matlab and validated against ANSYS predictions prior to use to ensure that a sufficient number of modes have been retained. The structural damping ratio was set in Matlab to $1 \%$ for all modes.

The FEM modal solution is computed with non-zero drive stiffness on the elevation and azimuth axes, and a negative stiffness included in Matlab to free these axes and obtain the unconstrained rigid body modes. Finite soil stiffness is included in the finite element model, and a similar procedure of subtracting the existing stiffness and inserting a desired stiffness can be used to explore the effects of different soils on the wind response.

Although the FEM is capable of modeling the individual segment support resonances, the support stiffness is set to a sufficiently high value so that the support resonances are not present in the modal solution. This reduces the number of modes obtained, and increases the prediction accuracy because the static contribution of the neglected modes is simply the segment support stiffness, which can be added separately in the model. The resonances themselves can be separately added for assessing achievable primary mirror control bandwidths as in Ref. 13. The 738 segment nodes are adequate to describe the low wavenumber deformation of the primary mirror that is relevant to seeing-limited image degradation, and it is not necessary to predict the rotations of the segments: this reduces the number of input degrees of freedom by nearly a factor of three. In addition to the normal forces on the primary mirror segments, 3 axis forces on M2 are included in the model, and the capability exists to also apply forces to M3 or Nasmyth platforms. The responses of all optical surfaces are used in computing the optical response.

The primary mirror actuators include a local displacement servo so that they accurately command the desired displacement at low frequencies. As noted, the dynamics associated with the segment support resonances do not affect the wind response of the telescope other than indirectly through limiting the achievable M1 control bandwidth. As a result, it is sufficient to include the support stiffness as a static correction, and model the actuators as perfect displacement actuators. While the M1 segment position control bandwidth is small compared to the achievable local servo loop bandwidth, this may not be true for M2 position control. In studying high bandwidth $(5-10 \mathrm{~Hz})$ optical guiding applied to M2 tip/tilt correction, the dynamics of the M2 actuator servo loop are relevant for predicting the closed loop behaviour of the guiding loop, and are included in the model. 

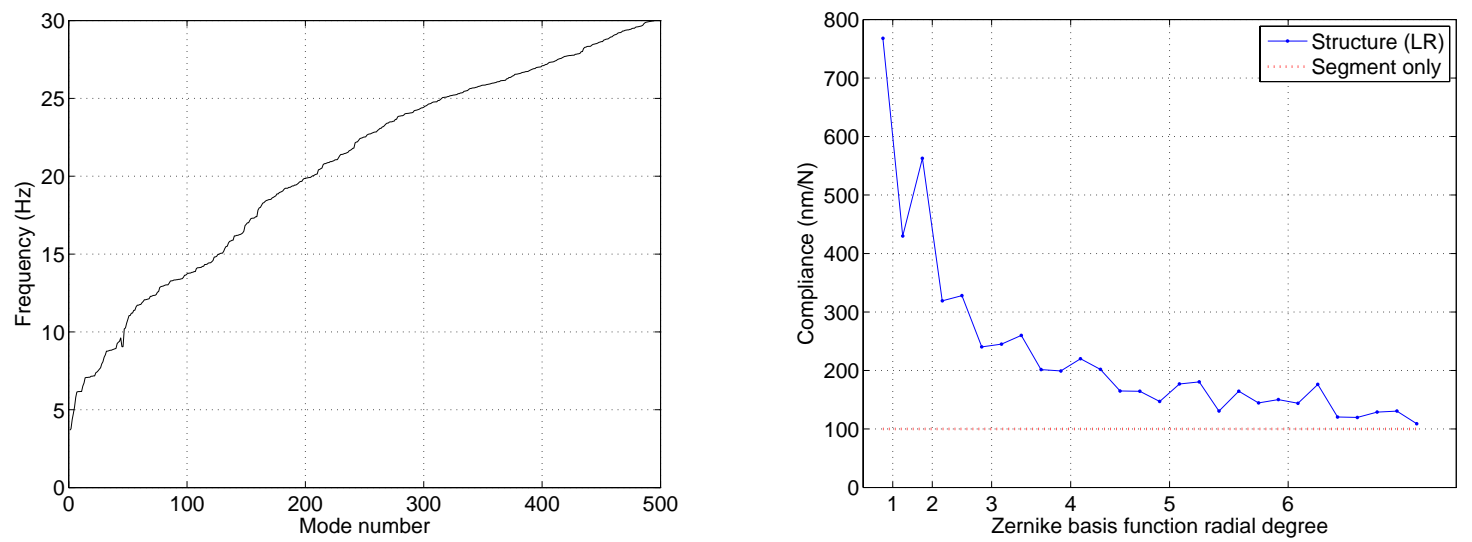

Figure 3. Relevant telescope structural characteristics: modal density (left) and primary mirror compliance (right). The static segment compliance of $100 \mathrm{~nm} / \mathrm{N}$ is dominant for sufficiently high wavenumber.
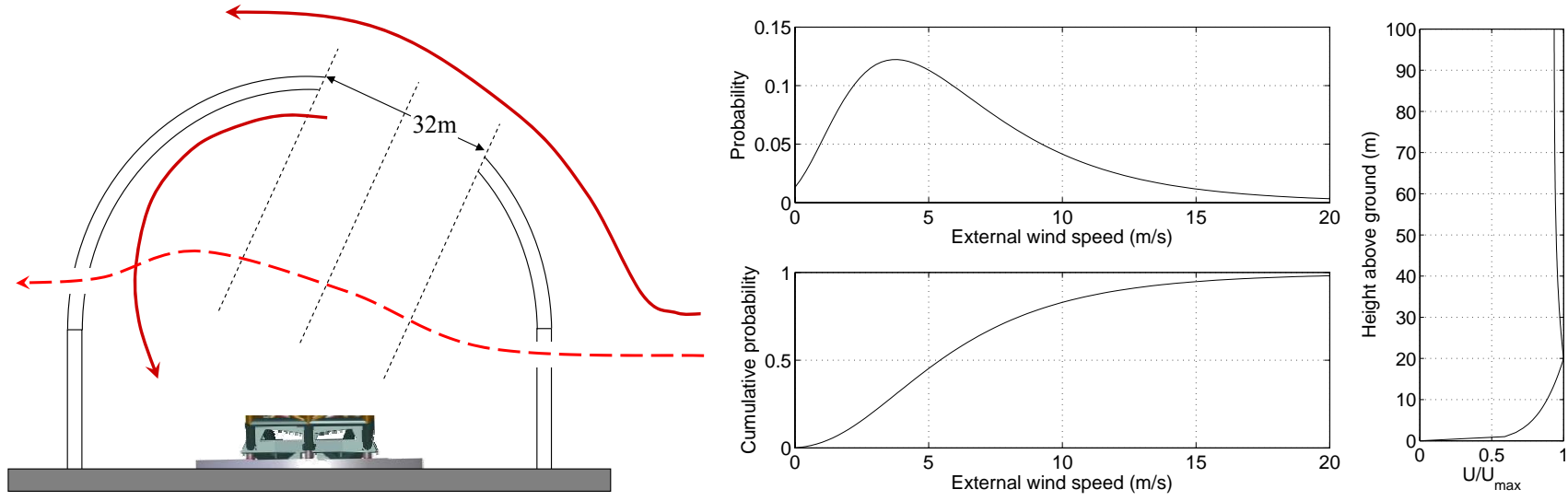

Figure 4. Schematic of wind mechanisms and flow patterns within the telescope enclosure (left), assumed external wind speed probability and cumulative probability distributions (center), and assumed external wind speed vertical profile (right).

\section{WIND}

Wind turbulence enters the telescope enclosure both through the viewing aperture, and through any vents that are chosen to be open in order to mitigate thermal seeing. The dominant turbulence inside the enclosure results from the flow passing over and through these openings, and not from the ingestion of outside turbulence. ${ }^{11}$ There can be relatively large wind speeds in the vicinity of the secondary mirror and its support structure, and with vents closed, an overall circulation within the enclosure that results in relatively low wind speeds near the primary mirror. With vents open, additional air flow passes over the primary mirror, and with the exception of high zenith angles, there is relatively little impact from the vents on the flow near M2. The flow patterns are shown schematically in Fig. 4. In addition to the broadband turbulence, it is possible to obtain tonal turbulence due to shear layer or Rossiter modes across the dome opening. ${ }^{8,11}$ While these are retained in the model, their amplitude is reduced by the presence of any open vents and they do not lead to any significant telescope motion.

The external wind speed is assumed to have a probability distribution given in Fig. 4, with a median wind speed of $5.5 \mathrm{~m} / \mathrm{s}$. Certain locations have higher wind than others, e.g. Mauna Kea median night-time wind speed at CFHT is $7 \mathrm{~m} / \mathrm{s}$. The figure shows a parameterized fit to measured data, collected at a sufficient height above ground to be above the boundary layer. The fit is scaled to give median speeds consistent with measurements taken at multiple mountaintop sites. The measured mountaintop wind speed profile is relatively insensitive to height above roughly $20 \mathrm{~m}$.

The parametric wind model represents our best available estimate of the wind loads on the telescope structure 
inside the enclosure, and is described in detail in Ref. 11. An earlier version of the wind model appears in Ref. 12. The information about the wind loads is integrated from three distinct complementary sources: full-scale velocity and pressure measurements obtained at Gemini South Observatory, ${ }^{5,6}$ spatial and temporal velocity data from wind-tunnel testing, ${ }^{8}$ and computational fluid dynamics (CFD). ${ }^{10}$ While full-scale measurements are in principle ideal, the TMT enclosure will be quite different from that at Gemini, and test conditions and measurement capabilities are unavoidably limited in full-scale tests. More information is available from limited test geometries in the wind tunnel. Finally, CFD provides the most comprehensive data as well as the ability to vary test geometries and conditions, although accurate simulations of unsteady 3-D turbulence is quite challenging.

Since details of the dome and telescope design that might influence wind loads are not yet finalized, nor is the final site selected, it is neither possible nor important to rely on every detail of the wind loads that can be measured in any of these data sources. Instead, it is important to focus on general trends and conclusions, and develop reliable estimates for representative wind loads while recognizing that locally significant wind loads may be possible on some areas of the primary mirror due to detailed characteristics of nearby structure, or that there may exist particular orientations of the telescope with respect to the wind that lead to much higher dynamic loads due to unusual orientation-specific flow patterns.

The wind model produces an estimate of the force on M2 (including the effective area of the supporting structure) and the distributed forces on M1 due to the turbulence generated by flow passing over and through the slit and flow through vents opened to mitigate thermal seeing. Loads on M3 or Nasmyth platforms could in principle be included but have not been simulated to date. We have chosen to compute pressure or force amplitudes by first estimating the reduction in velocity afforded by the protection from the telescope dome, and then converting to pressure or force through a local pressure coefficient or drag coefficient factor on the dynamic pressure, so

$$
p=C_{p}^{\prime} \frac{1}{2} \rho U_{\text {eff }}^{2} \quad F=A \cdot C_{D} \frac{1}{2} \rho U_{\text {eff }}^{2}
$$

The effective velocity $U_{\text {eff }}$ used in Eq. (1) is the local velocity near the relevant structure, and is selected so that Eq. (1) holds for the unsteady pressure or force. If the velocity is separated into mean and unsteady components as $U=\bar{u}+u^{\prime}$, the pressure depends on $U^{2}=\bar{u}^{2}+2 \bar{u} u^{\prime}+u^{\prime 2}$. Thus for example, if $\bar{u} \gg u^{\prime}$, the unsteady pressure can be computed from $U_{\text {eff }}=\left(2 \bar{u} u_{\mathrm{rms}}\right)^{1 / 2}$. More generally, for Gaussian $u^{\prime}, U_{\mathrm{eff}}=\left(\left(2 \bar{u} u_{\mathrm{rms}}\right)^{2}+2 u_{\mathrm{rms}}^{4}\right)^{1 / 4}$.

In addition to the amplitude, the performance depends on the temporal spectrum and spatial correlation characteristics. All of the available data reliably suggests that the broadband turbulent pressure spectra can be reasonably approximated by a von Karman spectrum with outer scale determined by the source of turbulence (dome opening or vent opening):

$$
\Phi(f) \propto \frac{0.7731 / f_{0}}{\left(1+\left(f / f_{0}\right)^{2}\right)^{7 / 6}}
$$

The finite correlation length is accounted for by assuming frozen turbulence (not entirely valid), which leads to a frequency-dependent attenuation factor on integrating pressure over any finite area. ${ }^{11,23}$ This approach is used both over the M2 support structure to obtain an equivalent effective force on M2 that matches the torque about the elevation axis, and for the forces on M1. We represent the forces over the primary mirror by projecting the pressure onto Zernike basis functions and computing the appropriate spectral amplitude and roll-off for each basis function. This straightforward procedure determines the spatial distribution of the turbulent pressure field with wavenumber, and follows directly from only the frozen turbulence and von Karman assumptions.

Two closely related issues can be raised regarding this wind model. First, the wind field over the primary mirror is much more complex than can be represented by a single spatially-averaged rms pressure value. However, although it is certainly more complex, the details of the complexity are not practically knowable because they depend on design details and particular wind orientations, and it is thus unrealistic to assume that any specific representation of the details (e.g. from CFD) is any more accurate than the model used here. Second, although this approach is reasonable for long length scale deformation of M1, the turbulence outer scale is assumed to be determined by the source of turbulence (the vent height or dome opening diameter) in an empty dome and the distribution of turbulence by length scale set by the frozen turbulence and von Karman assumptions. A real telescope structure will create more turbulence at smaller length scales than is predicted by the von Karman spectrum, and thus although the overall turbulence amplitude remains understood, the spatial distribution may be different from that predicted here. In particular, the current approach may lead to insufficient energy on 


\begin{tabular}{|c|c|c|c|}
\hline Parameter & Meaning & Value & Comment \\
\hline$U_{\infty}$ & External wind & $5.5 \mathrm{~m} / \mathrm{s}$ & Median wind across multiple sites \\
$\rho$ & Density & $0.82 \mathrm{~kg} / \mathrm{m}^{3}$ & $4000 \mathrm{~m}$ ASL \\
$A_{\mathrm{M} 2}$ & Transverse area of M2 & $10 \mathrm{~m}^{2}$ & Based on current design \\
$w_{\text {leg }}$ & Support leg depth & $0.6 \mathrm{~m}$ & Current design \\
$V_{\mathrm{M} 2 \text {,mean }}$ & Mean wind at M2 & $0.20 U_{\infty}$ & From WT, CFD, facing upstream \\
$V_{\mathrm{M} 2 \text {,eff }}$ & Wind at M2, effective & $0.25 U_{\infty}$ & From WT, CFD (see text) \\
$V_{\mathrm{M} 1 \mathrm{v}, \mathrm{mean}}$ & Wind at M1 with vents & $0.50 U_{\infty}$ & From CFD, max achievable \\
$V_{\mathrm{M} 1 \mathrm{v}, \text { eff }}$ & Wind at M1 with vents & $0.42 U_{\infty}$ & From CFD \\
$V_{\mathrm{M} 1 \mathrm{~s}, \mathrm{eff}}$ & Wind at M1, no vents & $0.07 U_{\infty}$ & From WT, Gemini \\
$D_{v}$ & Vent turb. outer scale & $10 \mathrm{~m}$ & Vent height \\
$D_{s}$ & Slit turb. outer scale & $32 \mathrm{~m}$ & Aperture diameter \\
$C_{D, \mathrm{M} 2}$ & Drag coeff., M2 & 1.5 & Conservative estimate \\
$C_{D, \text { leg }}$ & Drag coeff., leg & 2.0 & Flat plate, high aspect ratio \\
$C_{p, \text { slit }}$ & Local press. coeff., M1, slit & 1.0 & From Gemini \& CFD. \\
$C_{p, \text { vent }}$ & Pressure coeff., M1, vent & 0.5 & From Gemini \& CFD \\
\hline
\end{tabular}

Table 1. Nominal values for critical parameters that influence the wind loads (see text for more detailed descriptions).

\begin{tabular}{|l|c|c|c|c|}
\hline & $\begin{array}{c}\text { Velocity } \\
U_{\text {eff }}\end{array}$ & $\begin{array}{c}\text { rms Force or } \\
\text { pressure }\end{array}$ & $\begin{array}{c}\text { Corner } \\
\text { freq. } f_{0}\end{array}$ & $\begin{array}{c}\text { Torque about } \\
\text { Elev. axis }\end{array}$ \\
\hline M2 & $1.4 \mathrm{~m} / \mathrm{s}$ & $11.5 \mathrm{~N}$ & $0.034 \mathrm{~Hz}$ & $345 \mathrm{Nm}$ \\
Support legs & $0.8 \mathrm{~m} / \mathrm{s}$ & $5.5 \mathrm{~N}$ & $(.026, .05) \mathrm{Hz}$ & $165 \mathrm{Nm}$ \\
Combined & & $16.9 \mathrm{~N}$ & & $510 \mathrm{Nm}$ \\
\hline M1, vents closed & $0.4 \mathrm{~m} / \mathrm{s}$ & $0.06 \mathrm{~Pa}$ & $0.0125 \mathrm{~Hz}$ & $45 \mathrm{Nm}$ \\
M1, vents open $(1 \mathrm{~m} / \mathrm{s})$ & $0.84 \mathrm{~m} / \mathrm{s}$ & $0.2 \mathrm{~Pa}$ & $0.08 \mathrm{~Hz}$ & $125 \mathrm{Nm}$ \\
M1, vents open $(2 \mathrm{~m} / \mathrm{s})$ & $1.7 \mathrm{~m} / \mathrm{s}$ & $0.7 \mathrm{~Pa}$ & $0.16 \mathrm{~Hz}$ & $415 \mathrm{Nm}$ \\
\hline
\end{tabular}

Table 2. Summary of nominal forces and pressures on M1 and M2 for the nominal parameter values listed in Table 1. The distributed force on the three support legs are lumped into an equivalent force on M2. The pressure on M1 is described for vents closed and for vents opened sufficiently to produce a mean velocity of 1 or $2 \mathrm{~m} / \mathrm{s}$ across M1. For M1, the effective elevation axis torque is computed using the frozen turbulence assumption as described in the text.

short length scales that are relevant for predicting the differential segment motion that impacts high-contrast AO performance. A possible future strategy would be to introduce an additional factor $0<\gamma<1$ representing the fraction of turbulent energy that is redistributed to small length scales.

The key parameters used in the wind model are listed in Table 1. The external wind speed is of course variable, and the median value across multiple mountaintop sites is given here. The wind speed estimates at M1 and M2 are consistent between wind-tunnel testing and CFD of enclosures with minimized aperture area, and correspond to an upwind (worst-case) orientation. Drag coefficients are conservatively estimated, and the local pressure coefficient on the primary mirror is estimated conservatively from data collected at Gemini observatory in which both the nearby velocity and surface pressures were measured.

Using the parameter values in Table 1, the wind forces on M2 and the supporting structure for M2, and the pressure on M1 with and without vents open are given in Table 2. The forces scale quadratically with the external wind speed, so while this represents the median expected force, the mean force is somewhat higher. The corner frequencies scale linearly with external wind speed, and thus the response of the telescope structure will increase faster than quadratically as the external wind speed increases.

\section{OPTICAL CONSEQUENCES}

The performance metric for seeing-limited observations is quantified in terms of the optical path difference (OPD), computed from a linear optical model. ${ }^{18}$ This is projected onto Zernike basis functions. The performance can further be separated in terms of the image jitter, or tip/tilt components of the OPD ( $n=1$ radial degree) and image quality, composed of higher order OPD distortions $(n>1)$. 


\section{CONTROL}

The overall telescope involves many control loops, ${ }^{13}$ several of which have a significant impact on the wind response of the telescope. The most important in terms of image stability are the main axis drives, and the elevation drive in particular. In addition to local encoder feedback, the current design applies optical guiding at the mounts. Simulations have considered optical tip/tilt feedback to either M2 tip/tilt or directly to the mount drive systems in order to make this decision. The latter is constrained to a bandwidth below that of the mount control system, however, the conclusion from the model is that this bandwidth is sufficient. Other corrections of M2 position will be at a sufficiently low bandwidth to not significantly affect the wind response but only to compensate for gravity and thermal deformations.

Internal deformations of the primary mirror segment array are sensed by mechanical sensors that can measure all of the deformation pattern except for the global piston, tip and tilt of M1. ${ }^{19}$ Previous analysis indicates that the bandwidth of the M1 control loop is limited only by the segment support resonances, designed to be at $35 \mathrm{~Hz}$ or higher, and a $1 \mathrm{~Hz}$ control bandwidth is readily achievable. ${ }^{13}$ For simulation, we conservatively use a $0.5 \mathrm{~Hz}$ control bandwidth for M1. The sensor information can be used to accurately estimate local position errors ${ }^{20}$ and it is not necessary to transform in simulation from position to sensor measurements and back to positions other than to project out the unobservable global degrees of freedom.

The elevation axis open loop system is influenced predominantly by a zero at the locked-rotor resonance of $3.7 \mathrm{~Hz}$, and the first resonance at roughly $5 \mathrm{~Hz}$. The controller is proportional-integral-derivative (PID) feedback with a structural filter with $30 \mathrm{~dB} /$ decade rolloff and a notch filter. The first telescope structural mode is actively damped by the control (loop gain higher than one). The maximum achievable loop crossover frequency with this design is at $1.3 \mathrm{~Hz}$, limited by keeping a $6 \mathrm{~dB}$ gain margin for all flexible modes above $4 \mathrm{~Hz}$. A structural filter is included at $17 \mathrm{~Hz}$ with "modest" damping ratios, just enough to bring the peak at that frequency below $-6 \mathrm{~dB}$. This control design should be quite robust to variations in the resonance frequencies. Optical tip/tilt information passes through a transformation matrix to obtain position commands at the elevation and azimuth drives, and integral control is used.

The azimuth drive control has not yet been implemented in the model, however this is not expected to have a significant impact on performance except for the absence of optical guiding on the image motion orthogonal to the elevation axis. Simulations are conducted for a worst-case upwind orientation in which the wind forces on M2 do not excite the uncompensated direction of image motion.

\section{PERFORMANCE PREDICTIONS}

The wind, structure, optics, and controls models described earlier are combined into a single integrated model that predicts the optical consequences of the controlled structural response to parametric wind loads. The response is summarized below for median external wind conditions, for three categories:

- Wind on M2 causing deformation of M1:

This was a problem in some previously documented wind studies ${ }^{24}$ due to the coupling of the secondary support system through the primary mirror cell. For the TMT structure, the M2 support structure is directly connected to the elevation journals so loads on M2 do not significantly deform the primary mirror.

- Wind on M2 causing telescope rotation and M2 decenter:

As at existing observatories, forces on M2 do lead to image motion. The primary compliance is due to the finite bandwidth of the elevation-axis mount control system (the locked rotor deformation is small). However, the combination of (i) adequate shielding by the telescope enclosure and (ii) a design that minimizes the upper-end wind cross-section lead to relatively small median forces as noted in Table 1. Thus, the stiff TMT structure that allows a sufficiently high achievable control bandwidth leads to acceptable performance. This conclusion may not hold for sufficiently soft soil.

- Wind on M1 causing deformation of M1:

Without vents open to mitigate thermal seeing, the wind speed across M1 is small and the resulting deformations are small. If vents are opened to give 1-2 m/s across M1, the resulting image motion is higher but still acceptable. Low wavenumber response of M1 is readily controllable by M1 segment actuation so that the seeing-limited image blur is small. The high wavenumber response is dominated by the segment support compliance, and is not expected to be a problem for adaptive optics. 

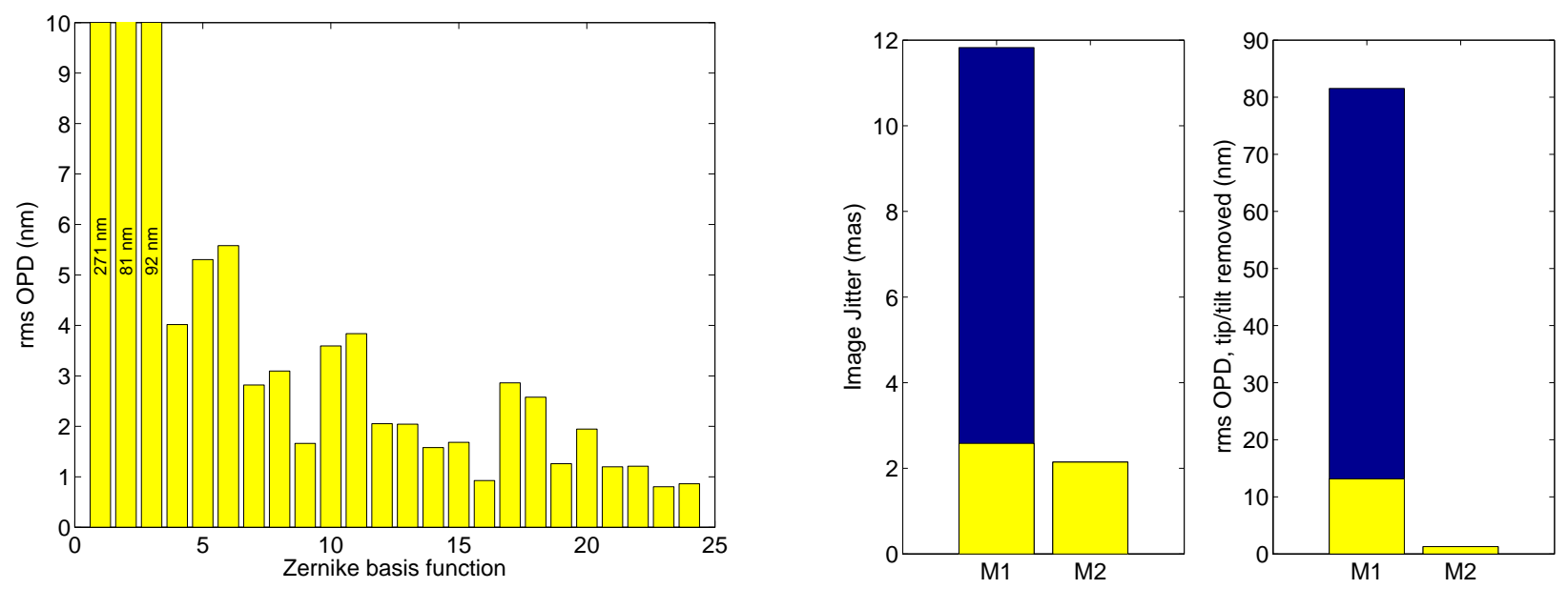

Figure 5. Zernike decomposition of OPD for nominal parameters (left); the axis is truncated to show the relative contributions of higher terms and the contributions to piston/tip and tilt are indicated with text. The image motion and tip/tilt removed OPD are shown on the right by disturbance source for a $2 \mathrm{~m} / \mathrm{s}$ wind on M1 (dark blue bar) and a $1 \mathrm{~m} / \mathrm{s}$ wind velocity (yellow).
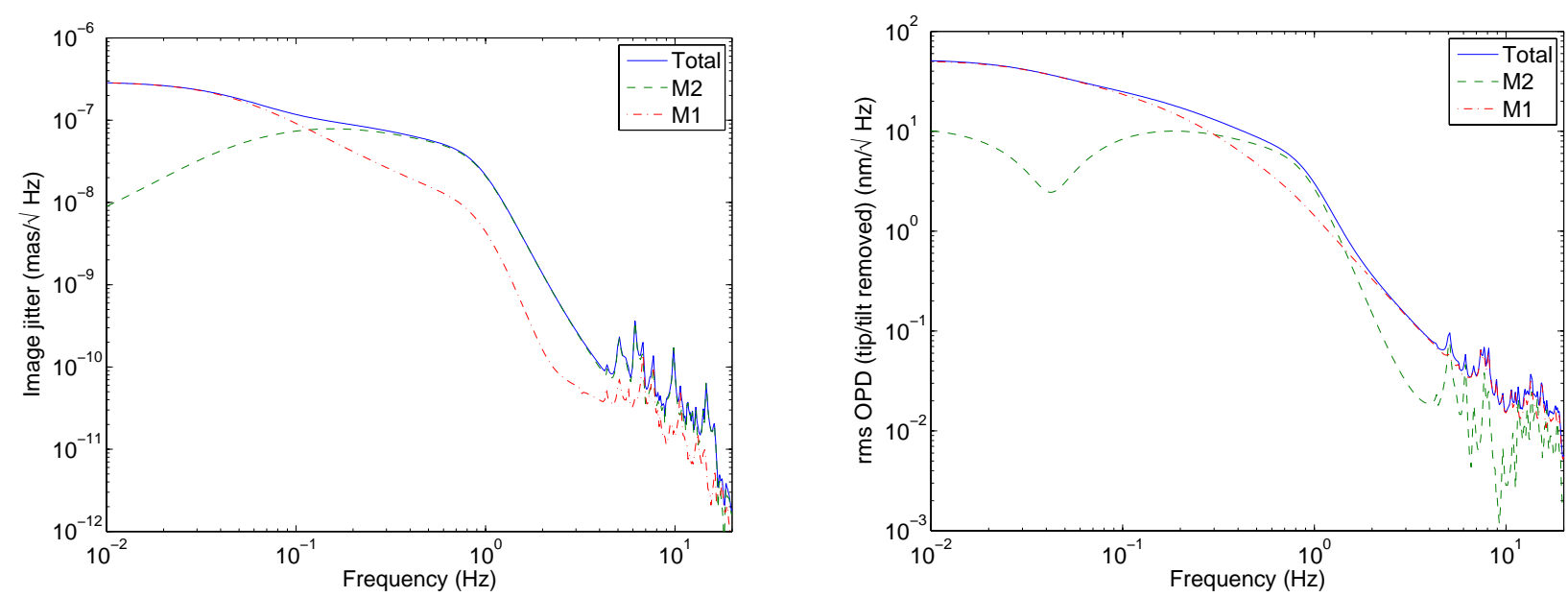

Figure 6. The spectrum of the image jitter (left) and tip/tilt removed OPD (right) for $1 \mathrm{~m} / \mathrm{s}$ velocity across M1. Each plot shows the overall spectru, the component due to M2 forces, and the component due to M1 forces.

The model predictions are separated into image motion (OPD tip/tilt, in milli-arcseconds (mas)) and higher order components (tip/tilt removed, or image blur). The response can further be separated into the component due to (i) forces on M2 and the supporting structure, and (ii) forces on M1. The nominal performance corresponds to the parameters listed in Table 1 (hence upwind viewing at median external wind conditions), with all relevant control loops closed, and vents opened to give $1 \mathrm{~m} / \mathrm{s}$ mean wind speed across M1. Variations in the M1 target wind speed are considered, with the vented area dependent on the target velocity and external wind speed.

The nominal image motion is 3.4 mas for a $1 \mathrm{~m} / \mathrm{s}$ velocity across M1, and 12 mas for a $2 \mathrm{~m} / \mathrm{s}$ velocity. The rms tip/tilt removed OPD for these two cases is 13 and $81 \mathrm{~nm}$ respectively. At median external wind speeds, the contribution to image motion due to forces on M1 and M2 are roughly comparable for $1 \mathrm{~m} / \mathrm{s}$ across M1, and dominated by the forces on M1 if the velocity is increased to $2 \mathrm{~m} / \mathrm{s}$ through venting. The tip/tilt removed OPD is dominated by the forces on M1 in either case. The nominal performance is illustrated graphically in Fig. 5, indicating the importance of the air flow required for mitigation of thermal seeing to the median wind-bufffeting performance. The Zernike decomposition of the nominal rms OPD is also shown in Fig. 5, illustrating a gradual decrease in energy with higher wavenumber, associated with both the decrease in input energy from the von 

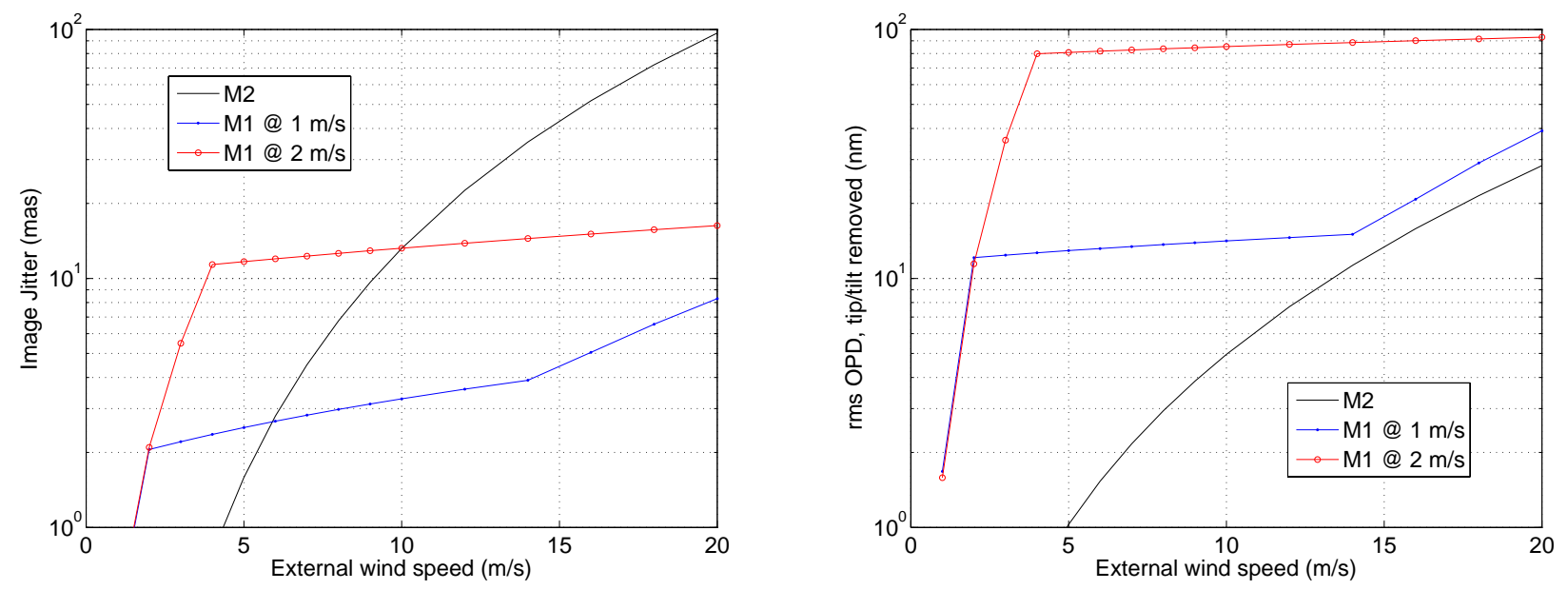

Figure 7. Parametric variation of image motion (left) and image blur (right) due to wind as a function of external wind speed (axis) and M1 target velocity.

Karman wind spectrum and the increase in structural stiffness illustrated in Fig. 3.

Fig. 6 shows the image motion and image blur spectra, and the spectra due to the M1 (at $1 \mathrm{~m} / \mathrm{s})$ and M2 forces. The $0.2 \mathrm{~Hz}$ corner frequency of the optical guide loop is evident in the image motion due to M2.

The variation in performance with external wind speed and the M1 target velocity design parameter is shown in Fig. 7. The performance information as a function of the target wind speed across M1 through vents will be combined with the results of thermal seeing studies ${ }^{15}$ to set a target for design of dome venting and the minimum achievable contribution to the error budget. The curves are obtained by setting the desired vent area as a function of external wind speed to obtain the desired target velocity. The sharp point in the curves at low external wind velocities corresponds to the point at which the vents are fully open and can no longer deliver the desired wind velocity on M1. At $14 \mathrm{~m} / \mathrm{s}$, the target velocity of $1 \mathrm{~m} / \mathrm{s}$ is achieved with the vents fully closed, and increases in external wind speed above this velocity lead to increased M1 wind speed.

One of the conclusions from the wind model is that the wind-buffeting response is acceptable for median external wind conditions. This is based on the dome design (ensuring low wind speeds near M2), structural design (minimizing cross-sectional area near M2) and control design. Three control loops contribute to the performance; the main drives and the elevation drive in particular, optical tip/tilt feedback to the drive control system, and the primary mirror segment actuation. Higher optical tip/tilt rejection would require that a high bandwidth tip/tilt capability be designed into M2 or M3. These simulation results illustrate that this is unnecessary. Higher bandwidth M1 control is likely possible, but is also not necessary. All of the performance results shown correspond to the worst-case (upwind) viewing orientation with respect to the wind, guaranteeing that windbuffeting is small regardless of orientation $50 \%$ of the time. At high external wind speeds, performance better than that shown in Fig. 7 may be achievable by pointing downwind.

\section{CONCLUSIONS AND FUTURE ENHANCEMENTS}

A model is presented of the optical consequences of wind-buffeting on a ground-based optical telescope inside an enclosure with the intent of addressing relevant design questions. The modeling strategy is chosen, consistent with the purpose, to facilitate rapid performance evaluation as a function of parameters and particular emphasis is placed on computational efficiency. This is distinct from designing a model to evaluate the performance of a single design with maximum fidelity. A parametric model of wind forces is applied to a finite-element derived structural dynamic model and coupled to a linear optical model, with control of the main telescope axes, primary mirror segments, and an optical guiding loop. Computations are performed in the frequency domain.

The primary conclusion from the model is that the wind response of the Thirty Meter Telescope for median wind conditions is acceptable without requiring additional effort on increasing control bandwidth. Since the response increases at least quadratically with increasing external wind speed, significant wind response is of course possible during some of the telescope operations. 
The model currently predicts image motion and low spatial wavenumber optical path differences relevant for seeing-limited operation of the telescope. These disturbances would be readily controllable by relatively low order adaptive optics systems. However, high contrast adaptive optics is unable to correct for inter-segment relative displacement differences. These differences are not well predicted by the current model, both because they are driven by small scale wind turbulence that is not practically predictable, and because the telescope response to these disturbances results primarily from segment support compliance and not from the overall telescope structural deformation. Therefore, the relevant modeling tools to address the adaptive optics relevant performance are quite different from those tools designed to address the seeing-limited performance metrics, and while the differential segment motion due to wind will be predicted by future analyses, different modeling tools will be developed to do so.

\section{Acknowledgements}

The authors gratefully acknowledge the support of the TMT partner institutions. They are the Association of Canadian Universities for Research in Astronomy (ACURA), the Association of Universities for Research in Astronomy (AURA), the California Institute of Technology and the University of California. This work was supported, as well, by the Canada Foundation for Innovation, the Gordon and Betty Moore Foundation, the National Optical Astronomy Observatory, which is operated by AURA under cooperative agreement with the National Science Foundation, the Ontario Ministry of Research and Innovation, and the National Research Council of Canada.

\section{REFERENCES}

1. Nelson, J. E. and Sanders, G. H., "TMT Status Report," Proc. SPIE Vol. 6267 Future Giant Telescopes IV, 2006. 6267-60.

2. Johns, M., Angel, R., Bernstein, R., Fabricant, D., McCarthy, P., Phillips, M., and Shectman, S., "Status of the Giant Magellan Telescope (GMT) project," SPIE 5489-28, 2004.

3. Andersen, T., Ardeberg, A., Riewaldt, H., Lastiwka, M., Quinlan, N., McNamara, K., Wang, X., Enmark, A., Owner-Petersen, M., Shearer, A., Fan, C., and Moraru, D., "Status of the Euro50 Project," SPIE, 2004.

4. Dierickx, P., Brunetto, E., Comeron, F., Gilmozzi, R., Gonte, F., Koch, F., le Louarn, M., Monnet, G., Spyromilio, J., Surdej, I., Verinaud, C., and Yaitskova, N., "OWL Phase A Status Report," Proc. SPIE Vol. 5489, Ground-Based Telescopes (Oschmann, J. M., ed.), 2004, pp. 391-406.

5. Cho, M. K., Stepp, L. M., Angeli, G. Z., and Smith, D. R., "Wind loading of large telescopes," Large Ground-Based Telescopes (Oschmann, and Stepp, eds.), 2002, pp. 352-367. Proc. SPIE 4837.

6. Angeli, G. Z., Cho, M. K., Sheehan, M., and Stepp, L. M., "Characterization of Wind Loading of Telescopes," Proc. SPIE Vol. 4757; Workshop on Integrated Modeling of Telescopes (Andersen, T., ed.), 2002, pp. 72-83.

7. Erm, T. M. and Angeli, G. Z., "TMT wind model validation with measurements on Keck and Gemini," SPIE 6271-27, 2006.

8. Pottebaum, T. and MacMynowski, D. G., "Buffeting of large telescopes: Wind tunnel measurements of the flow inside a generic enclosure," J. Fluids and Structures, Vol. 22, pp. 3-19, 2006.

9. Fitzsimmons, J., Dunn, J., Herriot, G., Jolissaint, L., Roberts, S., Mamou, M., and Cooper, K., "Predicting the aerodynamic performance of the Canadian Very Large Optical Telescope," Proc., SPIE 5497, Modeling and Systems Engineering for Astronomy (Craig, S. C., and M. J. Cullum, eds.), 2004, pp. 321-328.

10. Vogiatzis, K., Segurson, A., and Angeli, G. Z., "Estimating the effect of wind loading on Extremely Large Telescope performance using Computational Fluid Dynamics," Proc., SPIE 5497, Modeling and Systems Engineering for Astronomy (Craig, S. C., and M. J. Cullum, eds.), 2004, pp. 311-320.

11. MacMynowski, D. G., Vogiatzis, K., Angeli, G. Z., Fitzsimmons, J., and Nelson, J., "Wind Loads on Ground-Based Telescopes," submitted to Applied Optics, 2006. Early version available as TMT report TMT.SEN.TEC.05.008.REL01.

12. MacMynowski, D. G., Angeli, G. Z., Vogiatzis, K., Fitzsimmons, J., and Padin, S., "Parametric modeling and control of telescope wind-induced vibration," Proc., SPIE Vol. 5497, Modeling and Systems Engineering for Astronomy (Craig, S. C., and M. J. Cullum, eds.), 2004, pp. 266-277.

13. MacMynowski, D. G., Blaurock, C., and Angeli, G. Z., "Initial Control Results for the Thirty Meter Telescope," AIAA Guidance, Navigation and Control Conference, 2005. AIAA 2005-6075. 
14. Andersen, T., Enmark, A., Moraru, D., Fan, C., Owner-Petersen, M., Riewaldt, H., Browne, M., and Shearer, A., "A Parallel Integrated Model of the Euro50," SPIE 5497, 2004.

15. Vogiatzis, K. and Angeli, G. Z., "Strategies for Estimating Mirror and Dome Seeing for TMT," SPIE 6271-27, 2006.

16. Kerley, D., Roberts, S., Dunn, J., Stretch, N., Smith, M., Sun, S., Pazder, J., and Fitzsimmons, J., "Validation and verification of integrated model simulations of a thirty meter telescope," Proc. SPIE Vol. 5867 Optical Modeling and Performance Predictions II, 2005, pp. 220-231.

17. Angeli, G., Segurson, A., Upton, R., Gregory, B., and Cho, M., "Integrated modeling tools for large ground based optical telescopes," Proceedings of the SPIE, Vol. 5178, 2003, pp. 49-63.

18. Angeli, G. and Gregory, B., "Linear Optical model for a large ground based telescope," Proc. SPIE, Vol. 5178, 2003, pp. 64-73.

19. Chanan, G., MacMartin, D. G., Nelson, J., and Mast, T., "Control and Alignment of Segmented-Mirror Telescopes: Matrices, Modes, and Error Propagation," Applied Optics, Vol. 43, No. 6, pp. 1223-1232, 2004.

20. MacMartin, D. G. and Chanan, G., "Measurement accuracy in control of segmented-mirror telescopes," Applied Optics, Vol. 43, No. 3, pp. 608-615, 2004.

21. Halliday, D., Gedig, M., Brzezik, W., Zhou, Y., and Evans, P., "New approach to enclosure design for large telescopes," SPIE 4840-73, 2002.

22. Fitzsimmons, J., Sun, S., and Loewen, N. P., "Enclosure trade studies for the Thirty Meter Telescope," Proc. SPIE Vol. 6267 Future Giant Telescopes IV, 2006. 6267-70.

23. Padin, S., "Wind-induced Deformations in a Segmented Mirror," Applied Optics, Vol. 41, No. 13, pp. 23812389, 2002.

24. Padin, S. and Davison, W., "Model of image degradation due to wind buffeting on an extremely large telescope," Applied Optics, Vol. 43, No. 3, pp. 592-600, Jan. 2004. 